Poweld, E. O. (1958). J. gen. Microbiol. 18, 259-268

\title{
Criteria for the Growth of Contaminants and Mutants in Continuous Gulture
}

\author{
By E. O. POWELL \\ Microbiological Research Establishment, Ministry of Supply, Porton, Wiltshire
}

SUMMARY : Criteria are given for the establishment of growth of contaminants and mutants in continuous cultures of the Monod type. These explain, in particular, why contaminants so rarely interfere with the operation of such cultures.

In this paper I investigate the conditions under which the population of bacteria in a continuous culture of the Monod (1950) type may be displaced by foreign organisms, either mutants or contaminants. I have not attempted a full discussion of the balance between mutants and parent organisms, my object being mainly to analyse the danger of infection which besets the working of preparative cultures. The paper falls into two parts. The first concerns what may be called the biochemical criteria for successful displacement; in it the organisms are treated as chemical reagents, sensibly devoid of structure, and certain necessary conditions follow. The second concerns the statistical criteria which give the probabilities for successful displacement. I adopt the nomenclature and symbols of Herbert, Elsworth \& Telling (1956), in which the fundamental differential equations for conditions in the culture vessel are

where $t=$ time,

$$
\begin{gathered}
\frac{d x}{d t}=x\left(\mu_{m} \frac{s}{\boldsymbol{K}+s}-D\right), \\
\frac{d s}{d t}=\left(s_{R}-s\right) D-\frac{x}{\boldsymbol{Y}} \mu_{m} \frac{s}{K+s},
\end{gathered}
$$

$x$ = concentration of organisms by mass,

$s=$ concentration of limiting substrate,

$\mu_{m}=$ maximum mass growth-rate constant for the organism and medium concerned,

$\boldsymbol{Y}=$ yield constant for the given organism and substrate,

$K=$ saturation constant for the given organism and substrate,

$\boldsymbol{D}=$ dilution rate,

$s_{R}=$ concentration of limiting substrate in ingoing medium.

I assume throughout (i) that $D$ is held at a constant value, (ii) that before the entry of foreign organisms the culture has had time to reach a steady state, (iii) that the growth rate is limited by deficiency of a single nutrilite, (iv) that there is no interaction between two kinds of organisms but their competition for that nutrilite, ( $v$ ) that growth rates adjust themselves to changes in substrate concentration without appreciable lag, and (vi) that the mixing in the culture vessel is 'perfect', i.e. that the ingoing liquid is instantaneously and uniformly dispersed throughout the working volume. These restrictions 
simplify the analysis; they can be relaxed to some extent, but I do not think that a more general discussion would be of greater value in the present state of knowledge.

In many simple examples it is found that over a wide range of substrate concentration $(s)$ the growth-rate constant $(\mu)$ of the organism depends only on $s$. The form

$$
\mu(s)=\mu_{m} \frac{s}{\boldsymbol{K}+s},
$$

chosen in (1a), $(1 b)$ then appears to represent the relation between $\mu$ and $s$ with sufficient accuracy for practical purposes. ( $K$ is usually very small. Spicer (1955), takes $\mu \propto s$. This is approximately true in continuous cultures only when $D$ is not near its critical value

$$
D_{\mathrm{cr}}=\mu_{m} \frac{s_{R}}{K+s_{R}}
$$

unless $s_{R}$ is itself much less than $K$.) When a culture has been running for a long time (compared with $1 / D$ ) we have

$$
s+x / \boldsymbol{Y}=s_{R},
$$

i.e. the substrate concentration plus the mass concentration of organisms reckoned as substrate must equal the substrate concentration in the inflowing medium, whatever the initial values of $x, s$. We thus have ultimately

$$
\mu=\mu_{m} \frac{s_{R}-x / \boldsymbol{Y}}{\boldsymbol{K}+s_{R}-x / \overline{\boldsymbol{Y}}},
$$

and since $x$ must be positive $d \mu / d x$ is negative. Hence (Spicer, 1955) there is a final steady state with $x=\tilde{x}, s=\tilde{s}, d x / d t=0$ and

$$
\begin{gathered}
\tilde{s}+\tilde{x} / Y=s_{R}, \\
\mu=\mu_{m} \frac{\tilde{s}}{K+\tilde{s}}=D \quad\left(D<D_{\text {or }}\right) . \\
\left(\text { For } D \geqslant D_{\text {or }}, \tilde{x}=0, \tilde{s}=s_{R^{*}}\right)
\end{gathered}
$$

It can also be shown that $x$ and $s$ have each at most a single maximum or minimum for finite $t$, whatever the initial conditions.

\section{CHEMICAL KINETICS OF DISPLACEMENT}

Suppose the culture to be running at a steady rate with $x=\tilde{x}, s=\tilde{s}$. Let a foreign kind of organism - a contaminant or mutant of a single variety-be introduced at a concentration $y_{0}$. Let its saturation constant be $L$ and its maximum growth-rate constant $\lambda_{m}$. Then at the outset its concentration $y$ will increase at a rate

$$
\frac{d y}{d t}=y_{0}\left(\lambda_{m} \frac{\tilde{s}}{L+\tilde{s}}-D\right)
$$

For this expression to be positive, we must have

$$
\lambda_{m} \frac{\tilde{s}}{L+\tilde{s}}>D
$$


Eliminating $\tilde{s}$ between (4) and (5):

$$
\frac{\lambda_{m} K D}{\mu_{m} L-\bar{D}+D K}>D \text {. }
$$

Since we must have $D<\mu_{m}$ if $\tilde{x}>0$, the denominator on the left is positive and

$$
\lambda_{m} K-\mu_{m} L>D(K-L) \text {. }
$$

Hence, the concentration of the foreign organism can increase only if the following relation holds between $\lambda_{m}, \mu_{m}, K, L$ and $D$ :

$$
\left.\begin{array}{ll}
\frac{\lambda_{m} K-\mu_{m} L}{K-L}>D & (K>L), \\
\frac{\mu_{m} L-\lambda_{m} K}{L-K}<D & (L>K) .
\end{array}\right\}
$$

Or, if $\boldsymbol{D}$ is eliminated from (4) and (5),

$$
\left.\begin{array}{cc}
\frac{\lambda_{m} K-\mu_{m} L}{\mu_{m}-\lambda_{m}}>\tilde{s} & \left(\mu_{m}>\lambda_{m}\right), \\
\frac{\mu_{m} L-\lambda_{m} K}{\lambda_{m}-\mu_{m}}<\tilde{s} & \left(\lambda_{m}>\mu_{m}\right) .
\end{array}\right\}
$$

The survival of the foreign variety thus depends not only on $\lambda_{m}$ and $L$, but also on the level at which the culture is being run at the time of its appearance.

So far we have been concerned only with the first stages of the change in $y$. To discuss the final outcome when $(5),(6 a),(6 b)$ hold, we need the following information :

(i) At $t=0-, x=\tilde{x}$. The appearance of a mutant at concentration $y_{0}$ implies that at $t=0+, x=\tilde{x}-y_{0},=x_{0}$, say. If $Z$ is the yield constant for the new variety, we then have

and so if $Z>Y$

$$
\begin{aligned}
\tilde{s}+x_{0} / Y+y_{0} / Z & =\tilde{s}+x_{0} / \boldsymbol{Y}+y_{0} / \boldsymbol{Y}+y_{0}(1 / Z-1 / \boldsymbol{Y}) \\
& =\tilde{s}+\tilde{x} \boldsymbol{Y}+y_{0}(\mathbf{1} / Z-1 / \boldsymbol{Z}) \\
& =s_{R}+y_{0}(\mathbf{1} / Z-1 / \boldsymbol{Y}), \\
\tilde{s} & +x_{0} / \boldsymbol{Y}+y_{0} / Z<s_{R}, \\
\tilde{s} & +x_{0} / \boldsymbol{Y}+y_{0} / Z \geqslant s_{R^{*}}
\end{aligned}
$$$$
\text { and if } Z \leqslant Y
$$

If instead $y$ represents a contaminant-an addition to the population, not a replacement of part of it $-x_{0}=\tilde{x}$ and (8) holds in this case also.

(ii) At all subsequent times

$$
\frac{d y}{d t}=y\left(\lambda_{m} \frac{s}{L+s}-D\right)
$$

and (by an obvious extension of $(\mathbf{1} b)$ )

$$
\frac{d s}{d t}=\left(s_{R}-s\right) D-\frac{x}{Y} \mu_{m} \frac{s}{K+s}-\frac{y}{Z} \lambda_{m} \frac{s}{L+s} .
$$

Multiplying (1 $a$ ) by $1 / Y,(9)$ by $1 / Z$ and adding to (10):

$$
\frac{d}{d t}\left(s+\frac{x}{Y}+\frac{y}{Z}\right)=D\left\{s_{R}-\left(s+\frac{x}{Y}+\frac{y}{Z}\right)\right\},
$$


whence

$$
s+x / \boldsymbol{Y}+y / \boldsymbol{Z}=s_{R}+\boldsymbol{Q} e^{-D t},
$$

where the constant $Q$ is to be determined by the initial values of $s, x, y$. If (7) holds, $Q$ is negative; if ( 8 ) holds, it is zero or positive.

(iii) If $x, y, s$ have limits as $t \rightarrow \infty$, say $\tilde{x}_{1}, \tilde{y}_{1}, \tilde{s}_{1}$, they must simultaneously satisfy

$$
\begin{aligned}
& \tilde{x}_{1}\left(\mu_{m} \frac{\tilde{s}_{1}}{K+\tilde{s}_{1}}-D\right)=0, \\
& \tilde{y}_{1}\left(\lambda_{m} \frac{\tilde{s}_{1}}{L+\tilde{s}_{1}}-D\right)=0
\end{aligned}
$$

(from (1 $a$ ) and (9) by equating the derivatives to zero).

There are now two possibilities: (A) Equation (7) holds and $Q$ is negative in (11); (B) Equation (8) holds and $Q$ is zero or positive.

$$
\text { (A) At } t=0+\text { by (5) } \frac{d y}{d t}>0 \text {, }
$$

and so if $y$ does not approach a limit monotonically, the first extremum to occur must be a maximum. But for finite $t$

$$
\frac{d s}{d t}+\frac{1}{Y} \frac{d x}{d t}+\frac{1}{Z} \frac{d y}{d t}>0
$$

(from (11) with $Q$ negative). At an extremum of $y, d y / d t=0$ and $s$ has the value $\tilde{s}_{1}$ given by $(12 b)$. Comparison of $(12 b)$ with (5) shows that $\tilde{s}_{1}<s$, and $d x / d t<0$ (by (4)). Hence $d s / d t>0$. But

$$
\frac{d^{2} y}{d t^{2}}=\frac{d y}{d t}\left(\lambda_{m} \frac{s}{L+s}-D\right)+y \lambda_{m} \frac{L}{(L+s)^{2}} \frac{d s}{d t},>0,
$$

if $d y / d t=0, y>0$ and $d s / d t>0$; an extremum of $y$ for finite $t$ must be a minimum. Thus $y$ has no extremum for finite $t$, and by (13) it must approach a limit $\tilde{y}_{1}>y_{0}>0$. Then $d y / d t \rightarrow 0$ and $s \rightarrow \tilde{s}_{1},<\tilde{s} ;$ for large $t$

$$
\frac{1}{x} \frac{d x}{d t} \rightarrow\left(\mu_{m} \frac{\tilde{s}_{1}}{K+\tilde{s}_{1}}-D\right),<0 ;
$$

$d x / d t$ itself cannot remain finitely less than zero, since if it did, $x$ would tend to $-\infty$. So $x \rightarrow 0$, and the new variety entirely supersedes the old.

(B) When $Q$ in (11) is positive or zero

$$
\frac{d s}{d t}+\frac{1}{Y} \frac{d x}{d t}+\frac{1}{Z} \frac{d y}{d t} \leqslant 0
$$

If $x$ does not approach a limit monotonically, we have at every extremum (unless $x=0$ )

$$
s=\tilde{s} \text { and } d y / d t>0
$$

by (4) and (5), and hence $d s / d t<0$. From (1a),

$$
\frac{d^{2} x}{d t^{2}}=\frac{d x}{d t}\left(\mu_{m} \frac{s}{K+s}-D\right)+x \mu_{m} \frac{K}{(K+s)^{2}} \frac{d s}{d t},<0,
$$

if $x>0, d x / d t=0$ and $d s / d t<0$; every extremum of $x$ for finite $t$ is a maximum. Now at $t=0+, s=\tilde{s}$ and $d x / d t=0$, so the origin is a maximum of $x$, and there can be no other; $x$ approaches a limit $\tilde{x}_{1}<\tilde{x}$ monotonically. The limit must be 
zero, for if it were not, we should have $s \rightarrow \tilde{s}$ and $d y / d t>0$ again, and $d y / d t$ cannot remain finitely greater than zero. Thus $d x / d t \leqslant 0$ and so $s \leqslant \tilde{s}$ for all $t$;

$$
s+x / \boldsymbol{Y}<\tilde{s}+\tilde{x} / \boldsymbol{Y}=s_{\boldsymbol{R}} .
$$

Hence by (11)

$$
y>y_{0} \text {. }
$$

Since $y$ remains positive and $x \rightarrow 0$, we again reach a state where only one variety of organisms remains, and then continued oscillation is impossible.

Both (A) and (B) yield

and (11) and (12b) show that

$$
\begin{aligned}
& y \rightarrow \tilde{y}_{1}>0, \\
& x \rightarrow 0, \\
& s \rightarrow \tilde{s}_{1},
\end{aligned}
$$

$$
\begin{aligned}
\tilde{s}_{1} & =L D /\left(\lambda_{m}-D\right), \\
\tilde{s}_{1}+\tilde{y}_{1} / Z & =s_{R} .
\end{aligned}
$$

Analogous reasoning shows that if the sense of the inequalities (5), $(6 a),(6 b)$ is reversed, the outcome is

$$
\begin{aligned}
& y \rightarrow 0, \\
& x \rightarrow \tilde{x}, \\
& s \rightarrow \tilde{s},
\end{aligned}
$$

i.e. continued growth of the foreign variety cannot occur. However, a constant source of contamination by mutation or infection from without will give rise to a steady (but practically negligible) level of concentration of foreign organisms (Novick, 1955; Maxon, 1955), even though none is individually capable of sustained reproduction in the culture.

Finally if precisely

$$
\lambda_{m} \frac{\tilde{s}}{\boldsymbol{L}+\tilde{s}}=\mu_{m} \frac{\tilde{s}}{\boldsymbol{K}+\tilde{s}}=D,
$$

the two varieties can coexist indefinitely in any ratio of concentration; the outcome is

$$
\begin{aligned}
& y \rightarrow \tilde{y}_{1}>0, \\
& x \rightarrow \tilde{x}_{1}>0, \\
& s \rightarrow \tilde{s}_{1}=\tilde{s},
\end{aligned}
$$

but $\tilde{x}_{1}, \tilde{y}_{1}$ will depend on the initial values $x_{0}, y_{0}$. If the foreign variety is a contaminant, or if it is a mutant with $\lambda_{m} \neq \mu_{m}, L \neq K$, the probability of the occurrence of this condition is infinitesimal. In practice coexistence could only be maintained by constant adjustments of $D$; any deviation from strict equality would lead to one of the two previous results. It is also possible that there exist mutants having precisely the same growth characteristics as the parent variety (Novick \& Szilard, 1950). Then $\lambda_{m}=\mu_{m}, L=K$, and coexistence is possible at any dilution rate; continued formation of mutants of this kind may lead to their displacing the natives entirely.

With this last possible exception, equations $(5),(6 a),(6 b)$ are the only conditions which can lead to the successful growth of the foreign organisms, which then supersede the natives. 
Suppose, by way of example, that the established organism in a culture has $\mu_{m}=3 \mathrm{hr}^{-1}$ and that a mutant appears with $\lambda_{m}=2.5 \mathrm{hr}^{-1}$ and $L=\frac{3}{4} K$. Then

$$
\frac{\lambda_{m} K-\mu_{m} L}{K-L}=1
$$

If the culture is running with $D=0.5 \mathrm{hr}^{-1}$, by $(6 a)$ the mutant may establish itself. But if the dilution rate is $1.5 \mathrm{hr}^{-1}$, it cannot. Yet either kind of organism could survive alone at either dilution rate. The results of Novick \& Szilard (1950) indicate that such mutants, with $\lambda_{m} \leqslant \mu_{m}$ but $L<K$, do appear and can survive by reason of their faster growth at very low concentrations of limiting nutrilite.

The main restriction on the validity of the above reasoning is the assumption (iv) that the two kinds of organisms do not interact except in their competition for the limiting nutrilite; it is in this direction that the explanation of any Gegenbeispiel should first be sought. My colleague Mr R. Elsworth has experienced persistent contamination of Bacterium cloacae with Pseudomonas pyocyanea in continuous culture on a medium containing glucose, ammonia and sulphate as sole sources of carbon, nitrogen and sulphur. P. pyocyanea would scarcely grow alone on the medium. In this instance it is therefore likely that the products of metabolism (or lysis) of the native organisms encouraged the growth of the contaminants.

There is a simple general expression for the relation between $x, y$ and $t$ if (and only if) the two saturation constants are equal. We then have for all $t$

whence, eliminating $s$,

$$
\begin{aligned}
& \frac{d x}{d t}=x\left(\mu_{m} \frac{s}{K+s}-D\right), \\
& \frac{d y}{d t}=y\left(\lambda_{m} \frac{s}{K+s}-D\right),
\end{aligned}
$$

$$
\begin{gathered}
\frac{d}{d t}\left(\mu_{m} \log y-\lambda_{m} \log x\right)=D\left(\lambda_{m}-\mu_{m}\right) ; \\
\left(\frac{y}{y_{0}}\right)^{\mu_{m}} /\left(\frac{x}{x_{0}}\right)^{\lambda_{m}}=\exp \left\{\left(\lambda_{m}-\mu_{m}\right) D t\right\} .
\end{gathered}
$$

That is, if the two saturation constants are equal, the successful variety is the one with the larger maximum growth rate. By comparing (4) and (5) it can also be seen that if the maximum growth rates are the same, the successful variety is the one with the smaller saturation constant, although in this case no such simple relation as (14) exists. Both of these results are true for any possible $D$.

An equation, equivalent to (14), for the competition between species has been given by Volterra (1931).

\section{STATISTICS OF DISPLACEMENT}

The preceding section overlooks the circumstance that bacteria are discrete particles, and that normally only very few foreign organisms will enter the culture at one time. The chance that a few organisms will be washed out of the 
vessel before they divide is not negligible, and so the conditions we have established are necessary but not sufficient to secure the success of the incomers.

In what follows I shall assume that only a single organism of a mutant or contaminant appears at first in the culture. There is no difficulty in extending the reasoning to multiplets.

I have shown elsewhere (Powell, 1956) that the essential condition for the steady growth of an organism in terms of its generation-time distribution is

$$
\int_{0}^{\infty} e^{-D \tau} f_{D}(\tau) d \tau=\frac{1}{2}
$$

where $f_{D}(\tau)$ is the frequency function of generation time $(\tau)$ under the conditions obtaining in the culture vessel; $f_{D}(\tau)$ will depend on $\tilde{s}$ and so on $D$. This equation implies that the probability that an organism chosen at random will terminate in the vessel is $\frac{1}{2}$; or, alternatively, that the expected number of the immediate progeny of an organism is 1 ; consequently, that the number of organisms will remain constant. Suppose now that a single mutant or contaminant appears, and that the generation-time distribution of its kind is $g_{D}(\tau)$. Write $\epsilon$ for the probability that an organism of the new variety will terminate in the vessel:

$$
\int_{0}^{\infty} e^{-D \tau} g_{D}(\tau) d \tau=\epsilon
$$

Then we should expect the new variety to have a finite probability of multiplying successfully if $\epsilon>\frac{1}{2}$. If $\lambda$ is the growth-rate constant of the new variety:

we have

$$
\begin{gathered}
\lambda=\lambda_{m} \frac{\tilde{s}}{L+\tilde{s}}, \\
\int_{0}^{\infty} e^{-\lambda \tau} g_{D}(\tau) d \tau=\frac{1}{2},
\end{gathered}
$$

and comparison with (14) shows that $\epsilon>\frac{1}{2}$ implies $D<\lambda$ and so is tantamount to the inequality (5). The calculation of the chances is complicated by the existence of correlations between the generation times of related organisms. By far the most important is the correlation between sisters (about +0.6; Powell, 1955, 1956) and others are probably quite negligible in comparison. We need therefore to know the chance that one or both of a pair of sisters will terminate in the vessel. If $S_{D}\left(\tau_{1}, \tau_{2}\right)$ is the joint distribution of generation times for such pairs, the chance that both will terminate in the vessel is

$$
\int_{0}^{\infty} \int_{0}^{\infty} e^{-D\left(\tau_{1}+\tau_{2}\right)} S_{D}\left(\tau_{1}+\tau_{2}\right) d \tau_{1} d \tau_{2}=\eta^{2}, \text { say. }
$$

The chance that neither of a pair will terminate in the vessel is

$$
\int_{0}^{\infty} \int_{0}^{\infty}\left(1-\mathrm{e}^{-D_{\tau_{1}}}\right)\left(1-e^{-D_{\tau_{2}}}\right) S_{D}\left(\tau_{1}, \tau_{2}\right) d \tau_{1} d \tau_{2}=1-2 \epsilon+\eta^{2},
$$

since $S_{D}\left(\tau_{1}, \tau_{2}\right)$ may be assumed symmetrical in its arguments, and

$$
\int_{0}^{\infty} S_{D}\left(\tau_{1}, \tau\right) d \tau_{1}=\int_{0}^{\infty} S_{D}\left(\tau, \tau_{2}\right) d \tau_{2}=g_{D}(\tau)
$$


And the chance that some one terminates in the vessel and the other is washed out is

$$
1-\left(\eta^{2}+1-2 \epsilon+\eta^{2}\right)=2\left(\epsilon-\eta^{2}\right) \text {. }
$$

Now let $q_{n}$ be the chance that an organism of the new variety, if it does terminate in the vessel, has no progeny of the $n$th generation: $q_{1}=0$. This chance can be analysed into three components:

$$
q_{n}=A q_{n-1}^{2}+2 B q_{n-1}+C ;
$$

the first term is the chance that both daughter organisms terminate in the vessel but both fail to have progeny of the $(n-1)$ th generation; the second that only one terminates in the vessel, and has no progeny of the $(n-1)$ th generation; the third that neither terminates in the vessel. In our application, $A=\eta^{2}, B=\epsilon-\eta^{2}, C=1-2 \epsilon+\eta^{2}$, and these are exclusive and exhaustive, so

$$
q_{n}=\eta^{2} q_{n-1}^{2}+2\left(\epsilon-\eta^{2}\right) q_{n-1}+\left(1-2 \epsilon+\eta^{2}\right)
$$

We are interested in the probability that the foreign organism will still be represented in the vessel after many generation times; roughly, in $1-q_{\infty}$. It can be shown (see, for example, Feller, 1950) that the $q$ in equations such as (17) have a limit, here given by the smaller root of

$$
q_{\infty}=\eta^{2} q_{\infty}^{2}+2\left(\epsilon-\eta^{2}\right) q_{\infty}+\left(1-2 \epsilon+\eta^{2}\right) .
$$

Hence

$$
q_{\infty}=1 \text {, }
$$

or

$$
q_{\infty}=\left(1-2 \epsilon+\eta^{2}\right) / \eta^{2} \text {. }
$$

If $\epsilon \leqslant \frac{1}{2}$, the second solution gives $q_{\infty} \geqslant 1$ and only $q=1$ is admissible-the new variety is certain to fail. If $\epsilon>\frac{1}{2}$, the probability of success is given by the second solution, provided that the organism which first enters the vessel happens to terminate in it. Let us write $\epsilon_{i}$ for the chance that the foreign organism will terminate in the vessel. It may well happen that $\epsilon_{i} \neq \epsilon$, i.e. that the expectation for the first organism is not typical of its kind. For instance there may be a lag in the growth of an isolated contaminant or 'phenotypic delay' in a mutant (see, for example, Ryan \& Wainwright, 1954). Then the probability that the foreign organism will have progeny after many generations is

$$
\left.\begin{array}{rlr}
p_{\infty}=\epsilon_{i}\left(1-q_{\infty}\right) & =\epsilon_{i}\left(\frac{2 \epsilon-1}{\eta^{2}}\right) & \\
=0 & \left(\epsilon>\frac{1}{2}\right), \\
\left(\epsilon \leqslant \frac{1}{2}\right) .
\end{array}\right\}
$$

Of course, the growth of the new organism causes a change, very slight at first, in the cultural conditions and so in $\epsilon$ and $\eta$. But the number of native organisms is so large in practice that the new variety can multiply considerably without sensibly altering the conditions. By the time say 1000 organisms of the new variety are formed (if this ever occurs) the outcome is practically certain unless $\epsilon$ is only very little greater than $\frac{1}{2}$. For the probability of failure is then about $q_{\infty}^{500}$; if $\epsilon$ is as small as 0.505 , this is about $10^{-9}$. But after this stage the foreign organisms can be treated as varying continuously in concentration, by the method of the previous section. Success or failure is determined before multiplication has proceeded far enough to disturb the original equilibrium; equation (18) shows that a new variety may fail to establish itself, no matter how 
well it is adapted to do so, and if $\epsilon$ is only a little greater than $\frac{1}{2}$, the chance of success is poor. Some calculations based on measured generation times (Powell, 1956) suggest that $\eta$ will usually differ by no more than $2-3 \%$ from $\epsilon$.

Practical workers in this subject have commented on the infrequency of contamination; the above results accord with and explain this observation. But they also show that the fate of a mutant or contaminant is dependent on complex features of the growth process which often can only be known post facto; we cannot be sure that the mutability of a species is exhausted, nor legislate against unknown contaminants except by excluding them. It is, however, possible that effective precautions will be developed as experience increases; thus it may be possible to delay shut-down by an occasional temporary increase in dilution rate, or by injecting single doses of a bacteriostatic substance. As Maxon (1955) pointed out, a continuous culture on an industrial scale is economic only when it can be maintained for long periods.

The above reasoning relates to systems of continuous culture in which the dilution rate is fixed by the experimenter (Monod, 1950; Novick \& Szilard, 1950). Another type of apparatus (e.g. Bryson \& Szibalski, 1952) uses the optical density of the culture to control the dilution rate through a servo in such a way that the concentration of organisms remains constant. The chances for the first stages of growth of a foreign organism are the same in both systems. In the second, the subsequent behaviour depends on the yield constants and relative light-scattering power of the two varieties of organisms, and on the performance of the servo; moreover, $D$ is a function of $t$ during a period of changing population. The second system therefore requires a different form of treatment.

\section{SYNOPSIS}

Contaminant organisms or mutants which appear in a continuous culture can grow successfully only when their maximum growth rate and saturation constants stand in a certain relation to the constants of the native organisms and to the rate of flow through the culture vessel (equations $(5),(6 a),(6 b))$. If they do establish themselves, they displace the native organisms entirely. However, the foreign organisms or their progeny may happen to be washed out of the vessel, so that even when the necessary conditions are fulfilled, successful growth is still a matter of chance. The period of doubt is concentrated in the first few generations. The probability for success (equations (15), (16), (18)) depends on the distribution of generation times, and there are initial complications depending on the state of the foreign variety at the time of its appearance. If, under the conditions obtaining in the culture, the foreign variety has only a small advantage in growth rate over the natives, its chance of success is small.

Continued infection by contaminants or mutants of higher growth rate must lead to their ultimate success, but because the fate of any individual is uncertain they may not multiply appreciably until after a delay of indefinite length.

These results are true only under certain assumptions, of which the most restrictive is the postulate that there is no interaction between the two varieties of organisms other than their competition for the available nutrients. 


\section{REFERENCES}

Bryson, V. \& Szrbalski, W. (1952). Microbial Selection. Science, 11645.

Feller, W. (1950). Probability Theory and its Applications. New York: John Wiley and Sons.

Herbert, D., Elsworth, R. \& Telling, R. C. (1956). The continuous culture of bacteria: a theoretical and experimental study. J. gen. Microbiol. 14, 601.

Maxon, W. D. (1955). Continuous fermentation. Appl. Microbiol. 3, 110.

Monod, J. (1950). La technique de culture continue, théorie et applications. Ann. Inst. Pasteur, 79, 390.

Novick, A. (1955). Growth of bacteria. Annu. Rev. Microbiol. 9, 97.

Novick, A. \& Szilard, L. (1950). Experiments with the chemostat on spontaneous mutations of bacteria. Proc. nat. Acad. Sci., Wash. 36, 708.

Powell, E. O. (1955). Some features of the generation times of individual bacteria. Biometrika, 42, 16.

Powell, E. O. (1956). Growth rate and generation time of bacteria, with special reference to continuous culture. J. gen. Microbiol. 15, 492.

Ryan, F. J. \& Wainwright, L. K. (1954). Nuclear segregation and the growth of clones of spontaneous mutants of bacteria. J. gen. Microbiol. 11, 364 .

SPICER, C. C. (1955). The theory of bacterial constant growth apparatus. Biometrics, $11,225$.

Volterra, V. (1931). Leçons sur la théorie mathématique de la lutte pour la vie. Paris: Gauthier-Villars.

(Received 23 August 1957) 